TEMAS DE ACTUALIDAD

Rev Chil Salud Pública 2013 Vol 17 (1): 8-10

\section{¿Quién sabe realmente lo que está pasando en el colectivo? \\ Reflexiones a partir de un corte de agua potable}

\author{
Who ReAlly KNOWS What'S hapPening? Reflections on \\ A POTABLE WATER OUTAGE
}

El ejercicio de la Salud Pública -al igual que cualquier disciplina- se funda en un supuesto habitualmente invisible para sus practicantes; el del conocimiento privilegiado de éstos respecto de lo que podríamos llamar gruesamente lo que está pasando ${ }^{a}$ en su ámbito.

Sin duda, esta expresión se presta a controversias. La propia definición de aquello que está pasando implica que nosotros, observadores humanos -limitados a la comprensión de un conjunto restringido de variables, y limitados también por innumerables fuentes de incertidumbre en la construcción de dichas variables ${ }^{1}-$ debemos elegir qué pinceladas construirán la imagen de lo que está pasando.

Asumimos en forma inmediata, y sin cuestionarnos, que los practicantes profesionales de cualquier disciplina serán capaces de definir, de la mejor manera posible, el conjunto de variables necesarias y su organización. En nuestra vida cotidiana tendemos a dar eso por sentado, aun cuando seamos concientes, al menos por momentos, de las controversias que esto genera en nuestra propia disciplina.

Las características de la llamada web 2.0, donde cada persona adquiere la posibilidad de emitir señales plantea un serio cuestionamiento a esta asunción tácita. Si cada persona puede transformarse en esa perspectiva en un sensor de lo que ocurre ${ }^{2}$, cabe revisar nuestras asunciones tácitas sobre la forma en que conocemos eso que está pasando.

La consideración de un ejemplo local reciente puede darnos una idea del desafío que surge para la Salud Pública a partir de ello.

El 22 de enero de 2013, se produjo un corte de agua potable en Santiago, afectando a varios cientos de miles de viviendas. La Tercera online publicó ese día un mapa elaborado a partir de los datos entregados, vía twitter, por personas, desde distintos lugares de Santiago, respecto del estado del abastecimiento en su localización ${ }^{3 \mathrm{~b}}$.

La respuesta al requerimiento de saber lo que está pasando adquiere, esta vez, una forma diferente. Ya no se trata de un sistema basado en los datos que una burocracia termina concentrando en unos pocos privilegiados y que éstos "chorrean" a la población. La web 2.0 permite que cualquier persona puede organizar datos de libre disposición, de forma que resulten útiles a otros. Los conocedores privilegiados son sustituidos por

\footnotetext{
a. Utilizo intencionalmente esta expresión, que si bien puede resultar vaga, da cuenta de una necesidad fundamental de los seres vivos, accesible para cualquier lector con un pequeño ejercicio introspectivo. b. Accesible en http://goo.gl/maps/KKdC4.
} 
actores diversos, que pueden recuperar los datos que requieren de un sistema abierto, construyendo información pertinente a sus necesidades ${ }^{\mathrm{c}}$.

Constatamos algo que puede resultar de perogrullo, pero que debiera cambiar la forma en que vemos estos procesos:

El que sabe lo que está ocurriendo en un sistema es el sistema en su conjunto. Es el colectivo completo el que detenta el conocimiento de lo que está pasando y no un actor o actores privilegiados.

Es, entonces, la resultante de la acción de diversos agentes la que permite un acople de la operación del colectivo con lo que acontece, a través del acople con las situaciones particulares de cada escenario.

El mapa elaborado por Sebastián constituye una herramienta de lo que se conoce técnicamente como crowdfeeding $^{4}$; es decir, la puesta a disposición de datos a la población, con la posibilidad de que cada actor recupere los datos que le resultan pertinentes.

Prestemos atención a la falta de direccionamiento de los datos. En el modelo tradicional, las autoridades entregan los datos que ellas consideran pertinentes; incluso en ocasiones definen directamente lo que hay que hacer. Esto introduce el riesgo de desacople entre lo que las autoridades consideran pertinente y lo que la población requiere ${ }^{\mathrm{d}}$.

En un sistema basado en crowdfeeding, es cada actor quien define los datos que le resultan pertinentes, a partir de todos los que están disponibles. El mapa en cuestión constituye un avance signicativo también en el uso de datos provenientes de redes sociales. Es lo que llamamos crowdsourcing ${ }^{4}$. Si lo comparamos con lo ocurrido el 27 de febrero en la $\mathrm{ONEMI}^{5}$, advertimos la diferencia fundamental en cuanto a la confianza que se otorga a los datos provenientes de fuentes ajenas a la burocracia ${ }^{6,7}$.
Aunque no es el objetivo principal de dicho mapa, podemos comparar los resultados incluso desde el punto de vista de la imagen que pueden hacerse los que tradicionalmente se asumen como detentores de conocimiento privilegiado ${ }^{e}$.

$\mathrm{Al}$ momento de escribir este texto, a comienzos de febrero, el mapa contaba con 68.740 vistas y sorprendentemente, con sólo un comentario; una corrección a un dato.

Resulta pertinente mirar este trabajo práctico, aplicado a una situación nacional, a la luz de la experiencia internacional.

La red crisis mappers $^{\mathrm{f}}$ ha desarrollado un importante corpus teórico en sus webinars, caracterizando este proceso de conocimiento distribuido en cuatro fases:

\section{- Crowdsourcing,}

- Análisisg

- Visualización o despliegue de los datos

- Crowdfeeding

$\mathrm{Si}$ bien puede considerarse que el mapa del corte de agua da cuenta de todas ellas, presenta limitaciones asociadas a la forma en que se genera. Se trata de un mapa elaborado por el área digital de un medio de comunicación tradicional. Existe un operador del mapa que es quien carga los datos. Esto conlleva desde problemas de espacialización hasta vulnerabilidad de la actualización. Basta que el operador del mapa deba realizar otra tarea, para interrumpir su actualización y, por ende, la pertinencia de los datos.

Aunque prácticamente desconocidas en nuestro país, existen aplicaciones que permiten soportar estos sistemas de conocimiento distribuido de forma de reducir la dependencia de operadores.

Ushahidi ${ }^{i}$ permite ingresar en forma directa reportes de muy distintas fuentes; como encuestas generadas en la propia aplicación, SMS, Twitter, RSS de medios. Puede verse un ejemplo de utiliza-

c. Precisemos que en este texto se asume que los datos sólo configuran información cuando determinado actor, en determinada situación de tiempo y espacio, organiza dichos datos para resolver alguna incertidumbre.

d. Recordemos además, lo dicho previamente en cuanto a las dificultades para generar los datos que se pudiera considerar necesarios y la propia definición de este criterio.

e. Tengamos presente, de todas formas, la restricción de posicionamiento explicitada por el propio autor del mapa, al ser referidos los reportes a una localización de base comunal y no georreferenciados o geolocalizados.

f. www.crisismappers.net.

g. Es común denominar análisis a los procesos en que se ponen en común varios sets de datos, buscando entender lo que está pasando. Dado que no se trata de un ejercicio de dividir la realidad para entender sus componentes, parece más adecuado hablar, en este caso, de interpretación.

h. En términos prácticos, resulta difícil establecer una secuencia temporal entre el procesamiento de los datos para interpretarlos y su despliegue, ya que la espacialización -una de las técnicas de despliegue más usadas- e incluso, la representación tiempo/espacio son fundamentales para la emergencia de patrones.

i. www.ushahidi.com. Y la versión Crowdmap (www.crowdmap.com), que corre en el servidor de dicha organización. 
ción en una simulación con personal no entrenado en https://simulacionmagsp2012.crowdmap.com.

Los ejemplos presentados en la propia página de Ushahidi ${ }^{8}$ y las diversas formas de despliegue presentadas por Helena Puig? dan cuenta de una arrolladora tendencia a aprovechar el conocimiento distribuido para remover los cimientos del poder tradicional, que separan a los que saben de los que no saben.

Esto último representa un desafío enorme para la Salud Pública. Luego de los espectaculares logros en la lucha contra el germen -apoyada en un enfoque centrado en el conocimiento privilegiado e incluso en medidas de policía sanitariasus practicantes hemos encontrado dicultades para abordar las enfermedades sin germen.

Los intentos por cambiarle los estilos de vida a la población por otros más saludables se han mostrado notoriamente infructuosos. No es que no esté claro qué conductas podrían prolongar el tiempo de vida o reducir la ocurrencia de determinados efectos en una población. Lo que ha fallado es la manera de lograr que la gente haga lo que le debiera resultar más saludable.

La emergencia de estas nuevas herramientas permite plantearnos un enfoque alternativo a aquél donde la autoridad de salud intenta convencer a la población de hacer lo que considera adecuado.

Aplicaciones que favorecen la autonomía y erosionan las diferenciales de poder basadas en el privilegio de los datos pueden convertirse en poderosas herramientas para que los actores decidan más responsablemente.

Aunque una revisión de la situación actual en nuestro país demuestra un sorprendente retraso en la incorporación de estas tecnologías a la gestión pública y severas dificultades institucionales para beneficiarse de las ventajas de la mayor disponibilidad de datos ${ }^{10}$, se trata de un proceso inevitable. Los practicantes de la Salud Pública sólo tenemos dos opciones; explorar creativamente estas herramientas, asumiendo como algo positivo esta bajada del pedestal o mirar a la distancia cómo se desarrollan estos procesos.

Licanray, 10 de febrero de 2013

\section{REFERENCIAS}

1. Carvajal Bañados Y. Incertidumbre en la medición de defunciones: una aproximación. Universidad de Chile, Facultad de Medicina, Escuela de Salud Pública. Santiago, Chile; 2011. Disponible en: http:// www.tesis.uchile.cl/tesis/uchile/2011/me-carvajal_y/ pdfAmont/me-carvajal_y.pdf. Revisado 2012/09/15.

2. Laituri M, Kodrich K. On Line Disaster Response Community: People as Sensors of High Magnitude Disasters Using Internet GIS. Sensors. 2008;8(5):3037\$3055. Disponible en: http:// www.mdpi.com/1424-8220/8/5/3037. Revisado 2012/08/20.

3. Sebastian (username). El estado del suministro de agua; 2013. Disponible en: http://goo.gl/maps/ KKdC4. Revisado 2013/01/30.

4. Meier P. Introduction to Crisis Mapping; 2011. Crisis Mappers Webinar Series. Disponible en: https:/ /vimeo.com/23286160. Revisado 2012/10/08.

5. Tapia J. Registro en video de los hechos ocurridos en el Centro de Alerta Temprana el 27 de febrero de 2010; 2010. webpage Radio Bio bio. Disponible en: http://rbb.cl/2927. Revisado 2011/07/30.

6. Alvayay E. El Mando y Control en la Respuesta a Catástrofes de la Naturaleza: Una estructura organizacional en red para colaborar con agilidad. Barberis RB, editor. Centro de Investigaciones Navales y Marítimas; 2011.

7. Vivaceta A, Bustamante O, Huiriqueo C. Objeto presente, objeto ausente. Aprendiendo de la crisis en el CAT-ONEMI y su registro. En: II Congreso Latinoamericano y del Caribe sobre Salud Global; 2013.

8. Ushahidi. What is the Ushahidi platform. Disponible en: http://vimeo.com/7838030. Revisado 2012/09/20.

9. Puig H. Crisis Mapping Analytics; 2011. CrisisMappers Webinar Series. Disponible en: http:/ /youtu.be/GtLrzN3LA60. Revisado 2012/10/24.

10. Vivaceta A. Una Aproximación Etnográfica al Desarrollo de un Sistema de Gestión para Emergencias y Desastres en la comuna de Quilpué. Escuela de Salud Pública, Universidad de Chile. Santiago; 2012. Available from: http:// es.scribd.com/doc/129849028/2013-0311Version-ANT. 